\title{
Seeking the Bottleneck in the Process of Airport Security Based on Petri Theory
}

\author{
Shu Wang \\ North China Electric Power University, Beijing, 102206 \\ alanncepu@foxmail.com
}

Keywords: Bottleneck; Petri theory; GSPN model; Token

\begin{abstract}
With the continuous progress of society and the continuous popularization of aviation, airport security more and more attention by the relevant departments. The airline is also committed to providing travelers with an excellent flight experience, and they want to minimize travel time at the airport while maximizing security travel. For this reason, this paper first combs the process of passenger security, and then based on Petri theory, the establishment of GSPN model, the purpose is to identify the airport security in the control of passenger throughput bottlenecks.

In order to judge the bottleneck of security check, based on Petri theory, the use of HIPS software, the establishment of GSPN model found obstacles to reduce the security process time bottleneck for the following two: First, the identity check. The second is to carry out the millimeter wave personal inspection link. The model determines the position of the bottleneck by calculating the average number of tokens in the different security links. The average number of banks is the bottleneck where the library is located. In improving passenger throughput and waiting time variance, it is helpful to focus on improving bottlenecks.
\end{abstract}

\section{Introduction}

Airport security which is significantly essential to both people and country has been one of the world's concerns since the terrorist attacks in US on September 11, 2001. Airports have security checkpoints, where passengers and their baggage are screened for explosive and other dangerous items. All these are to prevent passengers from hijacking or destroying aircraft. Simultaneously, airlines also aiming to provide pleasant and convenient flight for passengers by minimizing the time they spend waiting in line at security checkpoints or waiting for their flight. Therefore, there's a need to find a balance between desires to maximize security while minimize inconvenience to passengers. This article briefly introduces the security process, and the establishment of security process model, the purpose is to identify the constraints of passenger throughput bottleneck.

\section{Clear the Purpose and Procedures of Security Check}

Security inspection is one of the contents of the port inspection, whose goal is to ensure safety for all passengers in the journey. Only passengers who pass security check can board.

Passengers randomly arrive at the checkpoint and wait in a queue until a security officer can inspect their identification and boarding documents. The passengers then move to a subsequent queue for an open screening line; depending on the anticipated activity level at the airport, more or less lines may be open. Once the passengers reach the front of this queue, they prepare all of their belongings for X-ray screening.

All of their belongings are moved by conveyor belt through an X-ray machine, where some items are flagged for additional search or screening by a security officer. Meanwhile the passengers process through either a millimeter wave scanner or metal detector. Passengers that fail this step receive a pat-down inspection by a security officer. The passengers then proceed to the conveyor belt on the other side of the X-ray scanner to collect their belongings and depart the checkpoint area.

Security procedures are as follows: 


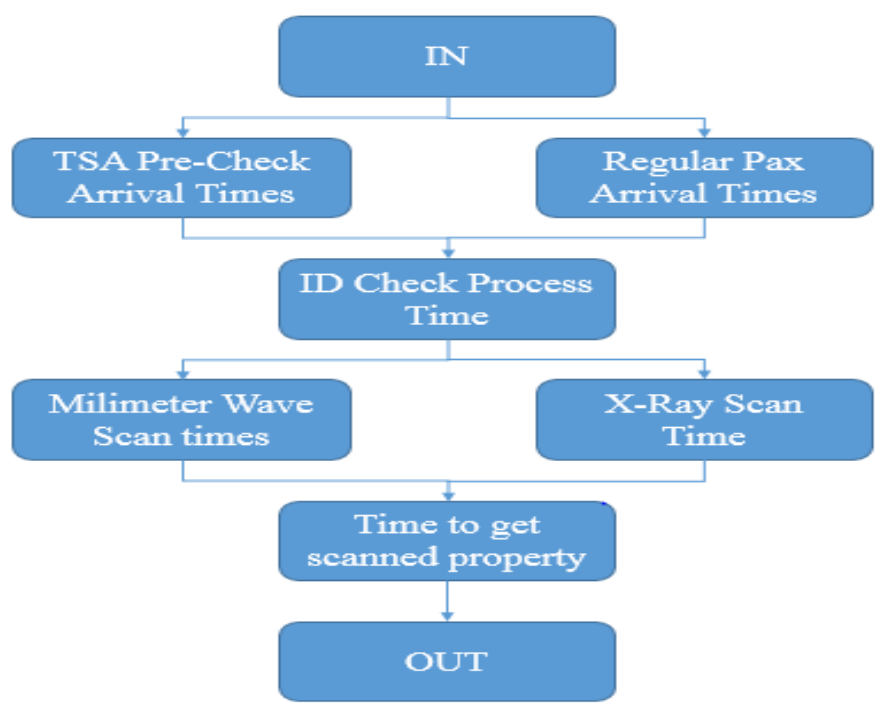

Figure 1

In the current security procedures, the bottleneck effect always impact efficiency extremely. When the passenger flow passes an entrance or exit, orderly queue can pass smoothly. The faster the speed is, the larger the flow of passenger flow is. However, a queue out of order will cause the reduction of passenger flow. In order to avoid the bottleneck effect, we need to build models to identify bottlenecks and try to optimize.

\section{Establish GSPN Model Based on Petri Theory and the Security Procedures}

Petri nets are a mathematical representation of discrete parallel systems, and a combination of graphs. The model can describe the process problem succinctly and concern the partial condition change as well as its influence on the entire system after the change. With the time parameters value given in attachment, we can regard the security procedures as a generalized stochastic Petri Net (GSPN) to build the model due to its synchronization, randomness.

We define a generalized stochastic Petri net model which is called, the parameters are as follows.

- a finite set of places;

- a finite set of transitions;

— the average instantaneous rate of change set of T;

- the relationship between places and transitions;

M0:— the initial identifier of the mapping network;

Place is used to represent the status of the activities prior to the security check, and transition is used to represent the security activities implemented in the various aspects of the process. According to the principle that the places and transitions alternately appear, connect the various security procedures in sequence and use the corresponding symbol to denote the place and transition.

Firstly, establish a basic single-channel security inspection process Petri net model according to the conditions in the subject by HIPS software. Assume that there is only one ID inspector and one X-ray machine. In this GSPN model, the time transition set for each time transition to determine an initiation rate $\lambda$, and. Instantaneous transition set. According to the condition, the probability of instantaneous transition $\mathrm{t} 1$ is $45 \%$, that means $\beta$ is $45 \%$, then the instantaneous transition probability of $\mathrm{t} 2$ is $1-\beta$, which is $55 \%$. The purpose of adding the time transition $t$ between the input place $i$ and the output place $o$ is to ensure continuity and strong connectivity of the GSPN model, whose initiation rate is $\lambda$. The model improved is as the figure shows: 


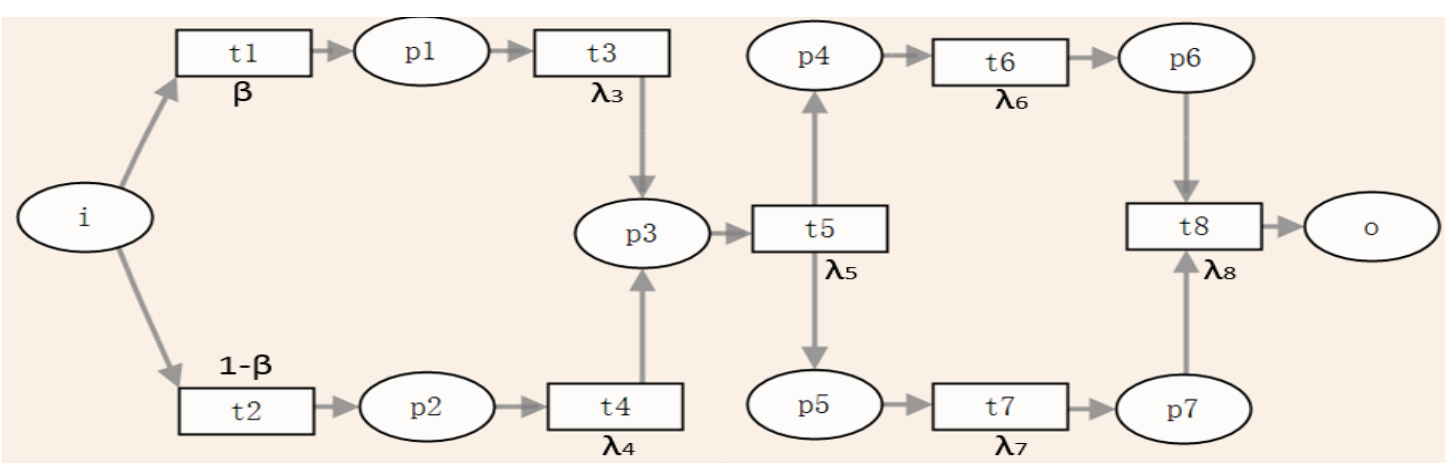

Figure 2

Description of symbols in the figure:

i: the input place, representing the passengers who have completed check-in, ready to accept security check.

p1: the place where passengers are ready in the Pre-Check lane.p2: the place where passengers are ready in the Regular Pax lane.p3: the place where passengers are ready for ID check.p4: the place where passengers have completed the ID check, ready for millimeter-wave screening.p5: the place where baggage are ready for X-ray screening.p6: the place where passengers have completed the millimeter wave screening.p7: the place where baggage have completed X-ray screening.

o: the output place, the passengers who have completed all security procedures.

$\mathrm{t} 1$ : the time point to prepare for the Pre-Check.

t2: the time point to prepare for the Regular Pax check.

t3: the time to do the Pre-Check;

t4: the time to do Regular Pax check;

t5: the time to do the ID check.

t6: the time to do the millimeter wave screening for passengers;

t7: the time to do X-ray scan for baggage;

t8: the time to get screened property.

\section{The Reliability Analysis of the Established Model}

According to the specific procedures of security, put a token at input place i multiple times randomly, then we will find that the token moves through every security check procedure, finally reaches the output place o. then we can prove that there is no dead change in the model, which illustrate its reliability.

\section{Solving the Value of Average Token}

According to the established GSPN model, draw the reachable identification table, and then draw the reachability map according to the table. The table is as follows:

Table 1

\begin{tabular}{|l|l|l|l|l|l|l|l|l|l|}
\hline & i & P1 & P2 & P3 & P4 & P5 & P6 & P7 & o \\
\hline M0 & 1 & 0 & 0 & 0 & 0 & 0 & 0 & 0 & 0 \\
\hline M1 & 0 & 1 & 1 & 0 & 0 & 0 & 0 & 0 & 0 \\
\hline M2 & 0 & 0 & 0 & 1 & 0 & 0 & 0 & 0 & 0 \\
\hline M3 & 0 & 0 & 0 & 0 & 1 & 1 & 0 & 0 & 0 \\
\hline M4 & 0 & 0 & 0 & 0 & 1 & 0 & 0 & 1 & 0 \\
\hline M5 & 0 & 0 & 0 & 0 & 0 & 1 & 1 & 0 & 0 \\
\hline M6 & 0 & 0 & 0 & 0 & 0 & 1 & 0 & 1 & 0 \\
\hline M7 & 0 & 0 & 0 & 0 & 0 & 0 & 0 & 0 & 1 \\
\hline
\end{tabular}

According to the table, draw the reachability map which is as follows. 


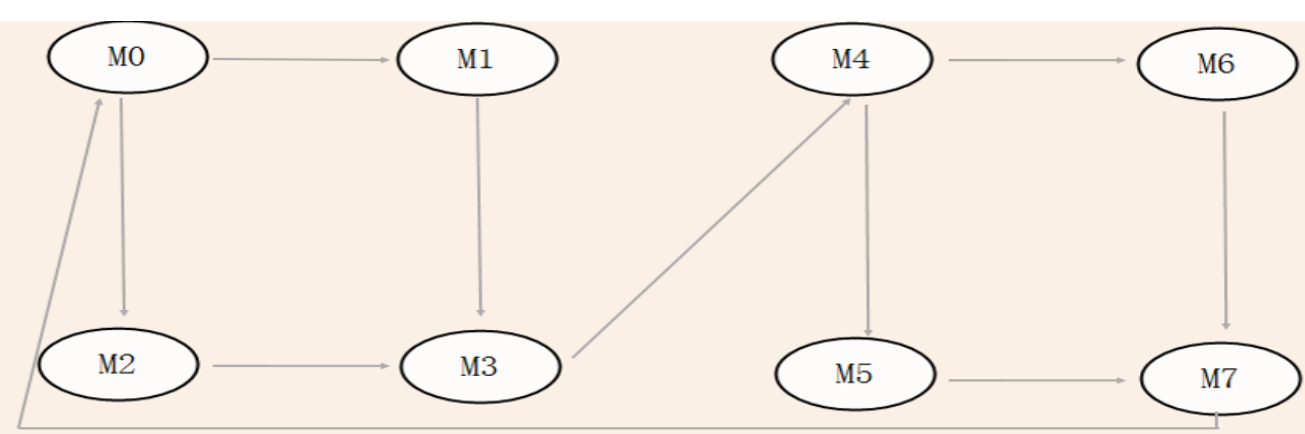

Figure 3

We can see that the reachability map is a Markov chain. After constructing the Markov chain which is isomorphic to the GSPN model, we begin to establish the density matrix to solve the steady state probability of Markov chain. Set the probability of being in each state is $\mathrm{X}=(\mathrm{x} 0$, $\mathrm{x} 1, \ldots, \mathrm{x} 7)$. According to the equation $\mathrm{XQ}=\mathrm{O}$, The probability distribution of the steady state can be obtained. And then determine the average number of tokens and change utilization rate of each place in the security procedures to identify bottlenecks.

From the data given in the problem, we can grasp the average time spent in each security procedure, to analyze based on the model.

Table 2

\begin{tabular}{|c|c|r|c|}
\hline Security procedure & time & ti & Symbolic meaning \\
\hline TSA Pre-Check Arrival Times & $04: 34.0$ & t3 & \\
\hline Regular Pax Arrival Times & $06: 17.7$ & t4 & \\
\hline ID Check Process Time & $00: 11.4$ & t5 & \\
\hline Milimeter Wave Scan times & $03: 52.4$ & t6 & \\
\hline X-Ray Scan Time & $00: 17.8$ & t7 & \\
\hline Time to get scanned property & $0: 28$ & t8 & \\
\hline
\end{tabular}

The number of tokens at each security procedure can be obtained from the model and the equation, which are as shown in the following table.

Table 3

\begin{tabular}{|c|c|c|c|c|c|}
\hline place & $\begin{array}{c}\text { The average } \\
\text { number of tokens }\end{array}$ & place & $\begin{array}{c}\text { The average } \\
\text { number of tokens }\end{array}$ & place & $\begin{array}{c}\text { The average } \\
\text { number of tokens }\end{array}$ \\
\hline $\mathrm{i}$ & 0.1496 & $\mathrm{p} 3$ & 0.0281 & $\mathrm{p} 6$ & 0.0174 \\
\hline $\mathrm{p} 1$ & 0.4635 & $\mathrm{p} 4$ & 0.2418 & $\mathrm{p} 7$ & 0.0107 \\
\hline $\mathrm{p} 2$ & 0.4594 & $\mathrm{p} 5$ & 0.1346 & $\mathrm{o}$ & 0.0429 \\
\hline
\end{tabular}

According to the average value of Token of each place, we can see, there are more tokens at p1, $\mathrm{p} 2, \mathrm{p} 4$. Therefore, these three places are more likely to form a bottleneck, in which $\mathrm{p} 1, \mathrm{p} 2$ represents the place where passengers are ready for the TSA Pre-Check or Regular Pax check, p4 is the place where passengers have been completed the ID check, ready for millimeter-wave scanning. The probable causes of bottlenecks form may be relatively larger number of passengers, lower efficiency of staff, insufficient number of security lanes, or some passengers need to undergo an additional security check.

\section{Conclusion}

Airport security needs to go through many processes, including millimeter wave detection, X-ray machines and so on. In the determination of bottlenecks, based on the security check process and Petri theory, the use of HIPS software for the establishment of the model. Draw the GSPN model. According to the GSPN model for the average number of analysis and calculation of the number of Token, we can see that in the access to the priority security channel and the general security channel, 
as well as the millimeter wave personal inspection location Tokor number more, so these three reservoirs easily formed bottleneck. So the airport can take relevant measures to solve these three locations stranded for too long a problem.

\section{References}

[1] Petri C. Communication with automata. Technical Report RADC-TR-65-377, Ro-me Air Dev[C]. Center, New York, NY, 1966: 1284-1291.

[2] Jiang X X, Zhou H, Cai B Q. Optimization Research on Process and Layout of Terminal Security Check [J]. Aeronautical Computing Technique, 2015.

[3] Jia Guoyang, Study on Optimization of Terminal Passenger Screening Service Process Based on LCIOWF,6,2015.

[4] GU Yang, ZHENG Min, ZHOU Hang, LI Yue, Research on Dynamic Allocations of Airport Security Check Resources ,9,2016.

[5] Wang Zhiqing, Research on the Convenient Engineering of Air Transportation and Its process optimization [D], 8, 2006.

[6] Yang Yun. Establishment and Optimization of Small and Medium - sized Airport Security Facilities.

[7] Jianguo X U. A Study on Work Pattern Problem for Airport Security Screening Officers [J]. Chinese Journal of Management, 2009, 6(8):1041-1046.

[8] http://baike.so.com/doc/9518926-9863012.html.

[9] Reisig W. Petri nets: an introduction [J]. Eatcs Monographs on Theoretical Computer Science, 1985.

[10] Ran N, Wang S, Su H, et al. Fault Diagnosis for Discrete Event Systems Modeled By Bounded Petri Nets[J]. Asian Journal of Control, 2017. 